\title{
INVARIANT MANIFOLDS OF HYPERCYCLIC VECTORS
}

\author{
PAUL S. BOURDON
}

(Communicated by Theodore Gamelin)

\begin{abstract}
We show that any hypercyclic operator on Hilbert space has a dense, invariant linear manifold consisting, except for zero, entirely of hypercyclic vectors.
\end{abstract}

Recently, Beauzamy [1-3] constructed examples of linear operators on Hilbert space having dense, invariant linear manifolds all of whose nonzero elements are hypercyclic. Using different techniques, Godefroy and Shapiro [8] showed how to construct such manifolds consisting of cyclic, supercyclic, or hypercyclic vectors. In this note, we show that any hypercyclic operator on a Hilbert space must have a dense, invariant linear manifold consisting, except for zero, entirely of hypercyclic vectors. Interest in constructing linear manifolds of cyclic/hypercyclic vectors arises from the invariant subspace/subset problem for linear operators on Hilbert space. If all of the nonzero vectors in a Hilbert space were, say, hypercyclic for an operator $T$, then $T$ would have no nontrivial, closed invariant subsets (and hence, no nontrivial invariant subspaces).

A vector $f$ in the (complex) Hilbert space $H$ is hypercyclic for the bounded linear operator $T: H \rightarrow H$ provided its orbit under $T$,

$$
\operatorname{Orb}(T, f):=\left\{f, T f, T^{2} f, \ldots\right\},
$$

is dense in $H$. If the set of scalar multiples of the elements of $\operatorname{Orb}(T, f)$ is dense in $H$, then $f$ is supercyclic for $T$; if the linear span of $\operatorname{Orb}(T, f)$ is dense in $H$, then $f$ is cyclic for $T$. A bounded linear operator $T$ on a Hilbert space is hypercyclic, supercyclic, or cyclic if it has, respectively, a hypercyclic, supercyclic, or cyclic vector. Hypercyclicity is a far more common phenomenon on Hilbert space than one might expect. For example, each of the following classes of linear maps contains hypercyclic operators: co-analytic Toeplitz operators [15, 8], compact perturbations of the identity [11, 6], translations [6], and composition operators $[4,5,14]$. Linear operators on finite-dimensional Hilbert space, however, are never hypercyclic, as the following proposition, due to Kitai [12], shows.

Proposition. Suppose that $T$ is hypercyclic on the Hilbert space $H$. Then the point spectrum of $T^{*}$ is empty.

Received by the editors November 4, 1991.

1991 Mathematics Subject Classification. Primary 47A15.

Research supported in part by the National Science Foundation (DMS 9023427).

(C) 1993 American Mathematical Society $0002-9939 / 93 \$ 1.00+\$ .25$ per page 
Proof. Suppose that $T^{*}$ has an eigenvalue $\lambda$ and that $g$ is a corresponding eigenvector. Let $f$ in $H$ be arbitrary, and let $\langle\cdot, \cdot\rangle$ denote the inner product of $H$. Then

$$
\left\{\left\langle g, T^{n} f\right\rangle: n=0,1,2, \ldots\right\}=\left\{\lambda^{n}\langle g, f\rangle: n=0,1,2, \ldots\right\}
$$

is not dense in $\mathbf{C}$. It follows that $f$ cannot be a hypercyclic vector; and because $f$ was arbitrary, $T$ cannot be hypercyclic.

Of course, the proposition above may be restated: If $T$ is hypercyclic, then $T-\lambda$ has dense range for any complex $\lambda$. That $T-\lambda$ has dense range whenever $T$ is hypercyclic is the key to the proof of the following.

Theorem. Suppose $T$ is hypercyclic on the Hilbert space $H$. Then there is a dense invariant linear manifold of $H$ consisting entirely, except for zero, of vectors that are hypercyclic for $T$.

Proof. Let $f$ be a hypercyclic vector for $T$. Following Godefroy and Shapiro [8], we note that

$$
M=\{p(T) f: p \text { is a polynomial }\}
$$

is a dense linear manifold of $H$ invariant under $T$ (dense, because $M$ contains the orbit of the hypercyclic vector $f$ ). We must show that every nonzero element of $M$ is hypercyclic for $T$.

Let $p(T) f$ be an arbitrary element of $M$. As pointed out in [8], $p(T) f$ will be hypercyclic- $\operatorname{Orb}(T, p(T) f)$ will be dense in $H$-provided $p(T)$ has dense range. To see this, observe that since $T$ commutes with $p(T)$,

$$
\operatorname{Orb}(T, p(T) f)=p(T) \operatorname{Orb}(T, f) ;
$$

that is, the orbit of $p(T) f$ under $T$ is the image of $\operatorname{Orb}(T, f)$ under the map $p(T)$. Now, if $p(T)$ has dense range then $\operatorname{Orb}(T, p(T) f)$ will be dense, being the image of the dense set $\operatorname{Orb}(T, f)$ under an operator with dense range. Thus, to establish the theorem it suffices to show that $p(T)$ had dense range.

Express $p$ as a product of linear factors; $p(T)$ is a scalar times a product of factors of the form $T-\lambda$. By the proposition, each of the factors $T-\lambda$ has dense range. Hence, $p(T)$ has dense range because it may be written as a product of operators with dense range.

A supercyclic operator need not have an invariant manifold all of whose nonzero elements are supercyclic (or cyclic). Let $T$ be hypercyclic on the Hilbert space $H(H \neq 0)$ and let $f$ be a hypercyclic vector for $T$. As pointed out in [10], $T \oplus I$ is a supercyclic operator on $H \oplus \mathbf{C}$ with supercyclic vector $f \oplus 1$ (the closure of the orbit of $f \oplus 1$ under $T \oplus I$ is $H \oplus 1$ and it follows that the closure of the set of scalar multiples of the elements of $\operatorname{Orb}(T \oplus I$, $f \oplus 1)$ is $H \oplus \mathbf{C})$. Suppose that $g \oplus \alpha$ is supercyclic or cyclic for $T \oplus I$. It easy to see that

$$
g \oplus \alpha-(T \oplus I)(g \oplus \alpha)=(g-T g) \oplus 0
$$

is a nonzero vector that is not cyclic for $T \oplus I$. Hence, any nonzero invariant linear manifold for $T \oplus I$ contains nonzero vectors that are not cyclic.

Remarks. 1. The results presented above are valid in a Banach space setting (with essentially the same proofs). 
2. The circle of ideas discussed in this paper yields a short, simple argument showing that the linear span of the collection of cyclic vectors of a cyclic operator is dense [13, Remark $4 ; 7 ; 9$, problem 166]. Suppose $T$ is a cyclic operator on a Hilbert (or Banach) space with cyclic vector $f$. Choose $\alpha$ large enough so that $T-\alpha$ is invertible (e.g., $\alpha>\|T\|$ ). Because $T$ commutes with $(T-\alpha)^{n},(T-\alpha)^{n} f$ is cyclic for $n=0,1,2, \ldots$. Hence the linear span of $\operatorname{Orb}(T-\alpha, f)$ is contained in the linear span of the cyclic vectors for $T$; moreover, the linear span of $\operatorname{Orb}(T-\alpha, f)$, being equal to the linear span of $\operatorname{Orb}(T, f)$, is dense.

\section{REFERENCES}

1. B. Beauzamy, Un opérator sur l'espace de Hilbert, dont tous les polynômes sont hypercyclic, C. R. Acad. Sci Paris Sér. I Math. 303 (1986), 923-927.

2. _ An operator on a separable Hilbert space, with many hypercyclic vectors, Studia Math. 87 (1988), 71-78.

3. _ An operator on a separable Hilbert space with all polynomials hypercyclic, Studia Math. 96 (1990), 81-90.

4. P. Bourdon and J. H. Shapiro, Cyclic composition operators on $\mathrm{H}^{2}$, Proc. Sympos. Pure Math., vol. 51, Amer. Math. Soc., Providence, RI, 1990, pp. 43-53.

5. Cyclic phenomena for composition operators (in preparation).

6. K. C. Chan and J. H. Shapiro, The cyclic behavior of translation operators on Hilbert spaces of entire functions, Indiana Univ. Math. J. 40 (1991), 1421-1449.

7. L. Gehér, Cyclic vectors of a cyclic operator span the space, Proc. Amer. Math. Soc. 33 (1972), 109-110.

8. G. Godefroy and J. H. Shapiro, Operators with dense, invariant, cyclic vector manifolds, J. Funct. Anal. 98 (1991), 229-269.

9. P. R. Halmos, A Hilbert space problem book, 2nd ed., Springer-Verlag, New York, 1982.

10. D. A. Herrero, Limits of hypercyclic and supercyclic operators, J. Funct. Anal. 99 (1991), 179-190.

11. D. A. Herrero and Z. Wang, Compact perturbations of hypercyclic and supercyclic operators, Indiana Univ. Math. J. 39 (1990), 819-830.

12. C. Kitai, Invariant closed sets for linear operators, Thesis, University of Toronto, 1982.

13. B. Sz.-Nagzy and C. Foiaş, Vecteurs cycliques et quasi-affinités, Studia Math. 31 (1968), 35-42.

14. M. Pavone, Chaotic composition operators on trees, Houston J. Math. 18 (1992), 47-56.

15. S. Rolewicz, On the orbits of elements, Studia Math. 32 (1969), 17-22.

Department of Mathematics, Washington and Lee University, Lexington, Virginia 24450

E-mail address: bourdon.p.s.@p9955.wlu.edu 